\title{
How long is the diagnosis of Familial Mediterranean Fever (FMF) delayed in a region where FMF is common in Turkey?
}

\author{
Y Karaaslan ${ }^{1,2^{*}}$, l Dogan ${ }^{3}$, A Omma $^{2}$, S Can Sandikci \\ From 8th International Congress of Familial Mediterranean Fever and Systemic Autoinflammatory Diseases \\ Dresden, Germany. 30 September - 3 October 2015
}

\section{Background and question}

Similar to other autoinflammatory diseases, diagnosis of FMF is often missed and markedly delayed, particularly when its prevalance is very low in a community. It's reported that diagnosis of FMF might be delayed more than 20 years. Although Turkey is one of the countries with a high prevalence of FMF, the diagnosis of the disease is markedly delayed in clinical practice.

In this study, we aimed to investigate the delay of diagnosis in patients followed up due to, or new diagnosed with FMF in the newly established Rheumatology Unit of a big tertiary medical center located in Çorum region. Çorum province is one of the region where FMF is very common in Turkey.

\section{Methods}

Consecutive 112 patients (38 male, 74 female) who admitted for follow up in the previous 3 months, fulfilling Tel-Hashomer FMF criteria were included in this study.

\section{Results}

The mean age of the patients was $32.9 \pm 11.7$ years (range 17-59, median 30), mean age at the onset of disease was $13.8 \pm 8.8$ years (range $2-40$, median 10 ), and the mean age at diagnosis was $25.7 \pm 12.8$ years (range $3-57$, median $23)$. The mean delay for diagnosis was found as $12 \pm 11.8$ years (range $0-49$, median 8 ). There was a delay of 10 years or more in $53.6 \%$ of the patients, and the delay for diagnosis was 20 years or longer in $11.6 \%$ of them.

Among the patients with a delay of diagnosis more than 10 years, before diagnosis of FMF, $11.7 \%$ of the patients were diagnosed with acute rheumatic fever,

\footnotetext{
${ }^{1}$ Hitit University Medical Faculty, Rheumatology, Çorum, Turkey
} Full list of author information is available at the end of the article
$5.0 \%$ of the patients were diagnosed with rheumatoid arthritis or juvenile chronic arthritis, $8.3 \%$ of the patients were diagnosed with spondylarthritis, and $10.0 \%$ of the patients were diagnosed with infection and $13.3 \%$ of the patients were diagnosed with other disease. $51.6 \%$ of the patients did not have any diagnosis.

There was abdominal pain in $92 \%$, fever in $87.5 \%$, arthritis in $35.7 \%$, chest pain in $21.4 \%$, erysipelas-like rash in $12.5 \%$ and history of an increase in acute phase reactants during attack in $97 \%$ of our FMF patients. Family history was positive in $65.4 \%$ of the patients, and $43.9 \%$ of them had history of surgery.

\section{Conclusions}

It is not difficult to diagnose FMF after onset of its clinical symptoms. But the diagnosis of FMF delayed for more than ten years above $50 \%$ of patients in Çorum region in Turkey. The most important reason for delay of FMF diagnosis in this region may be lack of "clinical suspicion" for the FMF among primary care and other physicians. Therefore measures must be taken to increase awareness of FMF in physicians and in community in this region.

\section{Authors' details \\ ${ }^{1}$ Hitit University Medical Faculty, Rheumatology, Çorum, Turkey. ${ }^{2}$ Ankara Numune Education and Research Hospital, Rheumatology, Ankara, Turkey. ${ }^{3}$ Çorum Education and Research Hospital, Rheumatology, Çorum, Turkey.}

Published: 28 September 2015

doi:10.1186/1546-0096-13-S1-P82

Cite this article as: Karaaslan et al:: How long is the diagnosis of Familial Mediterranean Fever (FMF) delayed in a region where FMF is common in Turkey? Pediatric Rheumatology 2015 13(Suppl 1):P82. 